\title{
Descriptive Characteristics of Males with Duchenne Muscular Dystrophy in Those Covered by Government (Medicaid) and Non-government (Private) Health Plans
}

\author{
Oscar Henry Mayer, ${ }^{1}$ John Karafilidis,${ }^{2}$ Kate Higgins ${ }^{3}$ and Brian Griffin ${ }^{3}$ \\ 1. Children's Hospital of Philadelphia, Philadelphia, PA, US; 2. Santhera Pharmaceuticals, Burlington, MA, US; 3. Truven Health Analytics,
} an IBM Company, Bethesda, MD, US.

$\mathrm{D}$ uchenne muscular dystrophy (DMD) is a rare, inherited neuromuscular disorder. Methods: To describe the clinical characteristics and healthcare resource utilization (HCRU) of male patients with DMD in commercial and Medicaid cohorts, this retrospective study identified male patients in the Truven Health MarketScan ${ }^{\oplus}$ Commercial and Medicaid databases diagnosed with hereditary progressive muscular dystrophy (HPMD) presumed to have DMD between 2011-2014. Patients with $\geq 2$ medical claims with a diagnosis for HPMD (International Classification of Diseases, 9th revision, Clinical Modification: 359.1) were included. Patients were followed for 12 months after diagnosis date, during which clinical characteristics and HCRU were assessed. Results: In total, 2,285 patients met the selection criteria. In these patients, corticosteroid and anti-infective agents were commonly utilized. Physician office visits were extremely common, with over $70 \%$ of all patients having at least one visit. Wheelchairs were commonly used, with the incidence of use increasing with age. Mechanical ventilators and airway clearance devices were underrepresented in the data. Conclusion: Patients with DMD had higher annual HCRU costs when compared with a non-DMD age-matched cohort, with patients in commercial cohorts having a higher annual average cost than those in Medicaid cohorts.

\section{Keywords}

Duchenne muscular dystrophy, cough assist device, administrative claims

Disclosures: Oscar H Mayer is a paid consultant for Santhera Pharmaceuticals. John Karafilidis is an employee of Santhera Pharmaceuticals. Kate Higgins and Brian Griffin are employees of Truven Health Analytics, an IBM company.

Review Process: Double-blind peer review.

Compliance with Ethics: This study involves a review of the literature and did not involve any studies with human or animal subjects performed by any of the authors.

Authorship: All named authors meet the International Committee of Medical Journal Editors (ICMJE) criteria for authorship of this manuscript, take responsibility for the integrity of the work as a whole, and have given final approval to the version to be published.

Open Access: This article is published under the Creative Commons Attribution Noncommercial License, which permits any noncommercial use, distribution, adaptation, and reproduction provided the original authors and source are given appropriate credit. (C) The Authors 2018

Received: April 17, 2018

Accepted: July 23, 2018

Citation: US Neurology. 2018;14(2):88-93

Corresponding Author: Brian Griffin

7700 Old Georgetown Rd, Suite 600, Bethesda,

MD 20814. E: briangri@us.ibm.com

Support: The publication of this article was supported by Santhera Pharmaceuticals (USA) Inc.
Duchenne muscular dystrophy (DMD) is a progressive, $\mathrm{X}$-linked recessive neuromuscular disorder, characterized by muscle weakness, wasting, and degeneration starting in early childhood. ${ }^{1,2}$ DMD is caused by a mutation in the dystrophin gene that leads to an absence of functional dystrophin protein. ${ }^{3}$ This disorder first affects muscles of the lower body, and progresses to the upper skeletal and cardiac muscles. ${ }^{1}$ In the US, the estimated prevalence is approximately 1.02 per 10,000 male persons, though as it is a rare disease, it may be underreported. ${ }^{4}$

The associated muscle weakening leads to increased disability, with initial symptoms presenting as difficulty walking, climbing stairs, and frequently falling. ${ }^{.}$DMD is incurable, and treatment is limited to supportive measures. Children with DMD will typically utilize corticosteroids, as these have been found to slow disease progression, and other support devices such as wheelchairs, ventilators, and cough assist devices., ${ }^{5,6}$ The real-world healthcare resource utilization of this condition is not well represented in the literature, and inclusion criteria for patients with DMD often differs, and sample sizes remain small. ${ }^{2,6}$ Further, a previous cost-of-illness study obtained data via patient survey, which is less accurate than administrative claims. ${ }^{8}$ This study aims to describe the healthcare resource use of patients with DMD in the commercial insurance and Medicaid populations, using a large administrative claims database.

\section{Methods}

Study sample selection

As a specific International Classification of Diseases, 9th revision, Clinical Modification (ICD-9-CM) for DMD is not available, the code 359.1 (hereditary progressive muscular dystrophy [HPMD]) was used. This code includes several other types of inherited muscular dystrophies (Appendix A), and the patient cohort was refined in several ways to further include only those presumed to be patients with DMD. Previous studies examining the cost of DMD used differing approaches for patient identification; Thayer et al. captured patients with diagnosis codes for both congenital hereditary muscular dystrophy and HPMD (ICD-9-CM: 359.0, 359.1), ${ }^{2}$ Miller et al. included patients diagnosed with HPMD and other possible cases from specialist clinics that may not have a formal DMD diagnosis. ${ }^{9}$ 


\begin{tabular}{|l|l|l|}
\hline Patient selection & Commercial N (\%) & Medicaid N (\%) \\
\hline $\begin{array}{l}\text { Patients diagnosed with hereditary progressive muscular dystrophy between September 30, } 2011 \text { and September 30, 2014* } \\
\text { (diagnosis date }=\text { first claim meeting all other criteria) }\end{array}$ & 5,986 & 3,288 \\
\hline And continuous enrollment in a health plan with medical and pharmacy benefits in 12 months following diagnosis date & $4,213(70.4)$ & $2,675(81.4)$ \\
\hline And male gender & $2,778(46.4)$ & $1,782(54.2)$ \\
\hline Total eligible patients & $1,137(19.0)$ & $1,148(34.9)$ \\
\hline Patients aged 6-10 years on diagnosis date & $300(5.0)$ & $313(9.5)$ \\
\hline Patients aged 11-14 years on diagnosis date & $248(4.1)$ & $284(8.6)$ \\
\hline Patients aged 15-18 years on diagnosis date & $277(4.6)$ & $260(7.9)$ \\
\hline Patients aged 19-25 years on diagnosis date & $320(5.3)$ & $296(9.0)$ \\
\hline
\end{tabular}

*To qualify, patients were required to have at least two medical claims, the first non-diagnostic, with a diagnosis of hereditary progressive muscular dystrophy (ICD-9-CM: 359.1), the second diagnosis occurring within 18 months of the first. ICD-9-CM = International Classification of Diseases, 9th revision, Clinical Modification; $N=$ number of patients.

In this study, patients were required to have at least two diagnoses for HPMD (ICD-9-CM: 359.1) between September 30, 2011 and September 30, 2014, and the diagnoses had to be within 18 months of each other. The first captured diagnosis could not be based on a laboratory claim, and patients were required to have at least 12 months of continuous health plan enrollment with pharmacy benefits following the date of the first DMD diagnosis. As DMD is the most common form of muscular dystrophy to affect children and because onset of disease is most often between the ages of three and five, ${ }^{1,6}$ patients aged 6-25 on their first captured diagnosis were included to assess disease-related costs across the full spectrum of patients with DMD; the cohort was restricted to males as DMD is an X-linked recessive condition. ${ }^{5}$

\section{Study design}

A retrospective, observational cohort design was used to describe male patients presumed to have DMD who were identified in an administrative claims database. From this cohort, patients were stratified based on age at first captured diagnosis of DMD during the study period, into mutually exclusive categories of males aged 6-10, 11-14, 15-18, and 19-25 years of age. Clinical characteristics, treatment patterns, and healthcare resource utilization were assessed in the 12 months following the diagnosis date. Average utilization and costs were calculated for the calendar year 2014 for a subset of the overall cohort with full benefit coverage during that calendar year. A control cohort was established of male patients of the same ages with commercial or Medicare insurance without a claim for DMD between September 30, 2011 and September 30, 2015, who have at least 12 months of continuous enrollment. The same characteristics and utilization was captured and compared to the patients with DMD.

\section{Data sources}

This study utilized two distinct US insurance claims data from the Truven Health MarketScan ${ }^{\circledR}$ Commercial Claims and Encounters and Medicare Supplemental Database and the Truven Health MarketScan ${ }^{\circledR}$ Multi-State Medicaid databases. Patient data sampled from the databases contain medical claims for healthcare services performed in the inpatient and outpatient settings, outpatient prescription drug claims, and enrollment data including demographic information and eligibility data. The commercial and Medicare databases contain administrative claims data for approximately 30 million covered lives in 2010-2014 from approximately 150 large employers and health plans across the US. The Multi-State Medicaid database provides access to comprehensive healthcare data on inpatient medical, outpatient medical and outpatient prescription drug claims for several million Medicaid beneficiaries annually (approximately 8.2 million patients in 2013 alone). All study data are de-identified and complied with all aspects of the Health Insurance Portability and Accountability Act of 1996 (HIPAA). As this study used only de-identified patient data, it was exempted from Institutional Review Board approval. Claims are fully-adjudicated and represent the full continuum of care across all settings of care. Diagnosis, drug and procedure codes on claims are used to define patient characteristics.

\section{Outcomes}

Clinical characteristics were captured in the 12-month follow up, including fractures, pneumonia, and sleep disorders (see Appendix B for full diagnosis code list). Use of anti-infective agents, corticosteroids, cough assist devices, oxygen supply, ventilation support, or wheelchairs were also captured. Medications were identified using National Drug Codes and Healthcare Common Procedure Coding System (HCPCS) codes, and medical devices were identified by Common Procedure Terminology codes, HCPCS codes, and ICD-9-CM procedural codes. It should be noted that this analysis did not include deflazacort (Emflaza ${ }^{\circledR}$, PTC Therapeutics, South Plainfield, NJ, US), as it was not approved for use in the US for the study period in question. Full generic medications included are listed in Appendix C.

Healthcare costs were captured over the 2014 calendar year, for a subset of those patients from the overall cohort that had complete benefit enrollment for that calendar year. Patients with an inpatient admission, any pharmacy claim, and any healthcare service were flagged, and costs were calculated as average payment among those who had a payment in that category.

\section{Results}

\section{Study sample characteristics}

A total of 2,285 patients were presumed to have DMD in our study sample, with 1,137 patients with commercial insurance and 1,148 patients enrolled in Medicaid (full attrition results in Table 1). In the commercial cohort, fractures were present in all DMD age groups; the highest percentage in the 15-18 age group, with $15.2 \%$ of patients having at least one medical claim in the year after qualifying diagnosis. Sleep issues were also seen in all cohorts and increased with age, from $10.3 \%$ of patients in the commercial 
Table 2: Clinical characteristics, commercial cohort

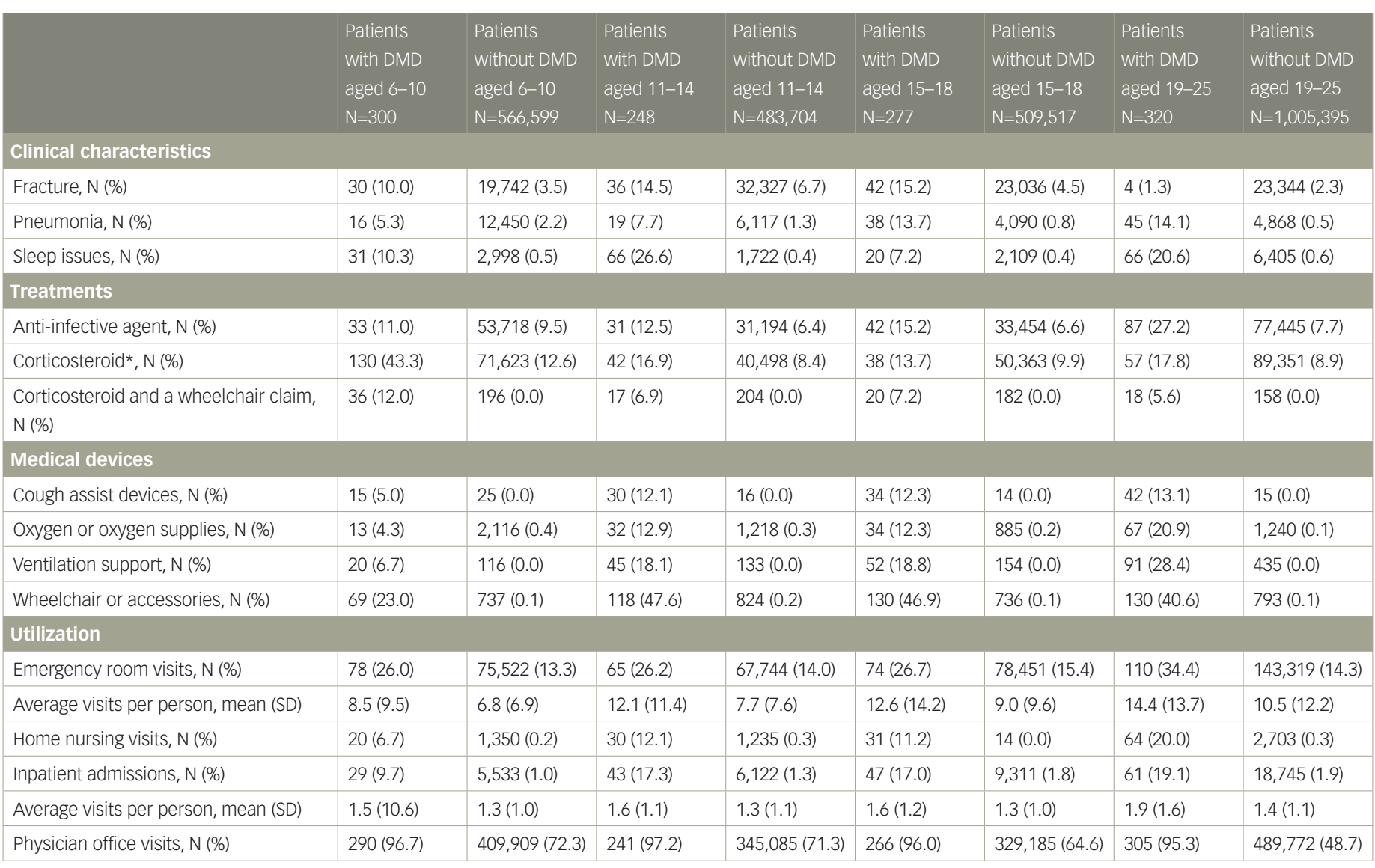

*Deflazacort is not currently available in the US through prescription pharmacies; this analysis does not include this medication. Full corticosteroid list can be found in Appendix C. Patient counts are reported as ' $N(\%)$ ', averages are reported as 'mean (SD)'. DMD = Duchenne muscular dystrophy; $N=$ number of patients; SD = standard deviation.

cohort 6-10 year age group and $20.6 \%$ of patients in the commercial cohort 19-25 year age group with DMD. Corticosteroid use was just over $43 \%$ in the youngest commercial cohort age group, but fell to $13.7 \%$ in the 15-18 age group. Anti-infective use was common among all DMD age cohorts, and increased from the 6-10 to 19-25 year age groups (11.0-27.2\% in the commercial cohort, 15.3-25.0\% in the Medicaid cohort).

At least $25 \%$ of patients in all age groups, in both the commercial cohort and Medicaid cohort, had at least one claim in the emergency department. In the Medicaid cohort, $73.3-85 \%$ of patients had a claim for a physician office visit, while home nursing services were utilized by a lower proportion of patients in the commercial cohort (6-20\% commercial versus 22-37\% Medicaid). Inpatient admissions were low and increased with age in both commercial and Medicaid cohorts, with $9.7 \%$ of patients aged 6-10 versus $19.1 \%$ of patients aged 19-25 in the commercial cohort having at least one inpatient admission with a diagnosis of DMD. The Medicaid cohort had similar findings, with $8.3 \%$ of patients aged $6-10$ versus $20.9 \%$ of patients aged 19-25. Healthcare service utilization was frequently higher for each category in the Medicaid cohorts than commercial cohorts.

All age cohorts of patients with DMD utilized medical devices in the year following first diagnosis date at a higher rate than the patients without DMD. Proportion of claims for wheelchairs or wheelchair accessories were similar in the commercial cohorts above age 10 , with $47.6 \%$ utilization in the
11-14 age group, $46.9 \%$ in the 15-18 age group, and $40.6 \%$ in the $19-25$ age group (versus $0.1-0.2 \%$ in the age-matched cohorts without DMD). Cough assist devices were not frequently seen, with the highest percentage of patients in the commercial cohort 19-25 age group with 13.1\%. Emergency department visit proportions were not significantly different between DMD and non-DMD cohorts, while inpatient admissions were significantly higher and increased with age (8.3\% with DMD versus $1.2 \%$ without DMD in the Medicaid cohort 6-10 age group, 20.9\% with DMD versus $4.4 \%$ without DMD in the 19-25 age group). Full commercial and Medicaid cohort's clinical characteristics are available in Tables 2 and 3.

\section{Costs}

Costs were calculated as an average over the 2014 calendar year, of those who had the specific service, and are reported as mean (standard deviation) in Table 4. Average pharmacy costs among patients with DMD remained fairly constant across commercial cohort age groups, ranging from $\$ 2,189(\$ 4,843)$ in the $15-18$ year age group to $\$ 2,868(\$ 9,123)$ in the 11-14 age group. Average annual total cost of all healthcare services among the commercial cohort were similarly high in the older age groups; the highest costs were seen in the 11-14 age group $(\$ 33,344$ [ $\$ 81,070])$, and lowest in 6-10 age group $(\$ 12,248[\$ 36,147])$. Similar trends were seen in Medicaid cohorts, with the highest annual cost seen in the 15-18 age group $(\$ 58,411$ [\$293,493]). Full commercial and Medicaid cohorts' utilization and expenditures are available in Table 4. 
Table 3: Clinical characteristics, Medicaid cohort

\begin{tabular}{|c|c|c|c|c|c|c|c|c|}
\hline & $\begin{array}{l}\text { Patients } \\
\text { with DMD } \\
\text { aged 6-10 } \\
\mathrm{N}=313\end{array}$ & $\begin{array}{l}\text { Patients } \\
\text { without DMD } \\
\text { aged 6-10 } \\
\mathrm{N}=167,648\end{array}$ & $\begin{array}{l}\text { Patients } \\
\text { with DMD } \\
\text { aged 11-14 } \\
\mathrm{N}=284\end{array}$ & $\begin{array}{l}\text { Patients } \\
\text { without DMD } \\
\text { aged 11-14 } \\
\mathrm{N}=122,583\end{array}$ & $\begin{array}{l}\text { Patients } \\
\text { with DMD } \\
\text { aged } 15-18 \\
\mathrm{~N}=260\end{array}$ & $\begin{array}{l}\text { Patients } \\
\text { without DMD } \\
\text { aged } 15-18 \\
\mathrm{~N}=154,422\end{array}$ & $\begin{array}{l}\text { Patients } \\
\text { with DMD } \\
\text { aged 19-25 } \\
\mathrm{N}=296\end{array}$ & $\begin{array}{l}\text { Patients } \\
\text { without DMD } \\
\text { aged 19-25 } \\
\mathrm{N}=59,581\end{array}$ \\
\hline \multicolumn{9}{|l|}{ Clinical characteristics } \\
\hline Fracture, N (\%) & $12(3.8)$ & 4,709 (2.8) & $26(9.2)$ & $6,452(5.3)$ & $19(7.3)$ & $6,802(4.4)$ & $9(3.0)$ & $1,864(3.1)$ \\
\hline Pneumonia, N (\%) & $22(7.0)$ & $2,426(1.4)$ & $33(11.6)$ & $915(0.7)$ & $42(16.2)$ & $1,020(0.7)$ & $40(13.5)$ & $583(1.0)$ \\
\hline Sleep issues, $N(\%)$ & $44(14.1)$ & $1,306(0.8)$ & $50(17.6)$ & $743(0.6)$ & $60(23.1)$ & $857(0.6)$ & $50(16.9)$ & $551(0.9)$ \\
\hline \multicolumn{9}{|l|}{ Treatments } \\
\hline Anti-infective agent, N (\%) & 48 (15.3) & $13,375(8.0)$ & $54(19.0)$ & $7,811(6.4)$ & $38(14.6)$ & $12,384(8.0)$ & $74(25.0)$ & $3,601(6.0)$ \\
\hline Corticosteroid*, N (\%) & $99(31.6)$ & $26,623(15.9)$ & $41(14.4)$ & $11,450(9.3)$ & $42(16.2)$ & $14,297(9.3)$ & $50(16.9)$ & $4,040(6.8)$ \\
\hline $\begin{array}{l}\text { Corticosteroid and a wheelchair claim, } \\
\mathrm{N}(\%)\end{array}$ & $30(9.6)$ & $76(0.0)$ & $20(7.0)$ & $44(0.0)$ & $27(10.4)$ & $64(0.0)$ & $16(5.4)$ & $49(0.1)$ \\
\hline \multicolumn{9}{|l|}{ Medical devices } \\
\hline Cough assist devices, $\mathrm{N}(\%)$ & $14(4.5)$ & $7(0.0)$ & $30(10.6)$ & $3(0.0)$ & $23(8.8)$ & $2(0.0)$ & $11(3.7)$ & $6(0.0)$ \\
\hline Oxygen or oxygen supplies, N (\%) & $17(5.4)$ & $435(0.3)$ & $26(9.2)$ & $179(0.1)$ & $38(14.6)$ & $135(0.1)$ & $60(20.3)$ & $90(0.2)$ \\
\hline Ventilation support, N (\%) & $25(8.0)$ & $44(0.0)$ & $36(12.7)$ & $37(0.0)$ & $48(18.5)$ & $46(0.0)$ & $74(25.0)$ & $78(0.1)$ \\
\hline Wheelchair or accessories, $\mathrm{N}(\%)$ & $93(29.7)$ & $276(0.2)$ & $141(49.6)$ & $238(0.2)$ & $133(51.2)$ & $295(0.2)$ & $108(36.5)$ & $242(0.4)$ \\
\hline \multicolumn{9}{|l|}{ Utilization } \\
\hline Emergency room visits, $\mathrm{N}(\%)$ & $113(36.1)$ & $42,653(25.4)$ & $102(35.9)$ & $30,229(24.7)$ & 95 (36.5) & $46,341(30.0)$ & $111(37.5)$ & $19,245(32.3)$ \\
\hline Average visits per person, mean (SD) & $8.0(8.0)$ & $6.8(6.8)$ & $11.3(12.4)$ & $0.6(3.0)$ & $13.8(16.0)$ & $1.4(4.7)$ & $24.4(40.5)$ & $16.8(27.2)$ \\
\hline Home nursing visits, $\mathrm{N}$ (\%) & $71(22.7)$ & $3,365(2.0)$ & $79(27.8)$ & 1,992 (1.6) & 97 (37.3) & $1,627(1.1)$ & $109(36.8)$ & $1,265(2.1)$ \\
\hline Inpatient admissions, N (\%) & $26(8.3)$ & $2,087(1.2)$ & $50(17.6)$ & $2,050(1.7)$ & $52(20.0)$ & $3,743(2.4)$ & $62(20.9)$ & $2,611(4.4)$ \\
\hline Average visits per person, mean (SD) & $1.7(1.1)$ & $1.3(1.0)$ & $1.7(1.7)$ & $1.3(1.1)$ & $1.8(1.3)$ & $1.4(1.4)$ & $2.1(1.9)$ & $1.9(2.0)$ \\
\hline Physician office visits, N (\%) & $266(85.0)$ & $96,938(57.8)$ & $234(82.4)$ & $70,553(57.6)$ & $208(80.0)$ & $76,498(49.5)$ & 217 (73.3) & $17,812(29.9)$ \\
\hline
\end{tabular}

*Deflazacort is not currently available in the US through prescription pharmacies; this analysis does not include this medication. Full corticosteroid list can be found in Appendix C. Patient counts are reported as ' $N(\%)$ ', averages are reported as 'mean (SD)'. DMD = Duchenne muscular dystrophy; $N=$ number of patients; SD = standard deviation.

\section{Discussion}

DMD is a rare, $\mathrm{X}$-linked recessive disorder that disproportionately affects males. ${ }^{4}$ Disease burden can be accelerated as physical symptoms begin as young as 3 years of age. As DMD is incurable, affected patients frequently utilize medical devices to assist in disease management, as well as supportive medication such as corticosteroids and anti-infective agents. Corticosteroids have been found to maintain muscle strength and prolong ambulation, even preserving upper limb strength after loss of ambulation. ${ }^{2}$ This manuscript sought to describe utilization practices and disease morbidity based on a review of a large medical database. A number of trends were seen and are described below.

As expected, many patients were on corticosteroids, the utilization on which declined with age after 13 years of age, roughly corresponding with the age at which most patients lose ambulation. Corticosteroids were seen in a high percentage of patients aged $6-10$ (43.3\% and $31.6 \%$, with commercial or Medicaid coverage, respectively) and declined in ages 11-14 and 19-25 (16.9\% and $17.8 \%$ in commercial, $14.4 \%$ and $16.9 \%$ in Medicaid aged $11-14$ and 19-25 respectively). Of note, as deflazacort had not been approved for use by the US Food and Drug Administration, it was not included in the list of corticosteroids.

Airway clearance is of critical importance to patients with DMD, particularly in advanced disease stages when respiratory muscles become compromised. ${ }^{12,13}$ Though intubation is necessary in a majority of patients with DMD in time, use of mechanical percussive devices is effective in delaying this step. ${ }^{14-16}$ In this analysis, we found documented utilization of mechanical cough assist devices in database claims to be surprisingly low. The reasons for this may be four-fold: first, this study captured utilization claims for 1 year after the patient's first captured diagnosis within our study period; it is possible that patients obtained a cough assist device following a previous, uncaptured DMD diagnosis if said diagnosis was outside our timeframe; second, as previously mentioned, our data is composed of administrative medical and pharmacy claims, if patients do not obtain their cough assist device through their insurance carrier, it will not be captured in our analysis; third, physician variability in prescribing practices may influence utilization of devices among patients; fourth, there may be data coding limitations we are not aware of that confines our analysis. Other literature sources describing utilization of cough assist devices are scarce, as respiratory care for patients with neuromuscular disease has improved dramatically over the last decade. The few studies that examine ventilation use rates are limited to single-institution studies and rely on retrospective medical chart analysis, as is seen in McKim et al. 2013. ${ }^{16}$ This underrepresentation of cough assist devices in the data highlights the need for continued research, especially given the updated speed at which data are now available, which can assist in assessing the full burden of disease in patients with DMD in the real-world. 
Table 4: Utilization and expenditure, 2014

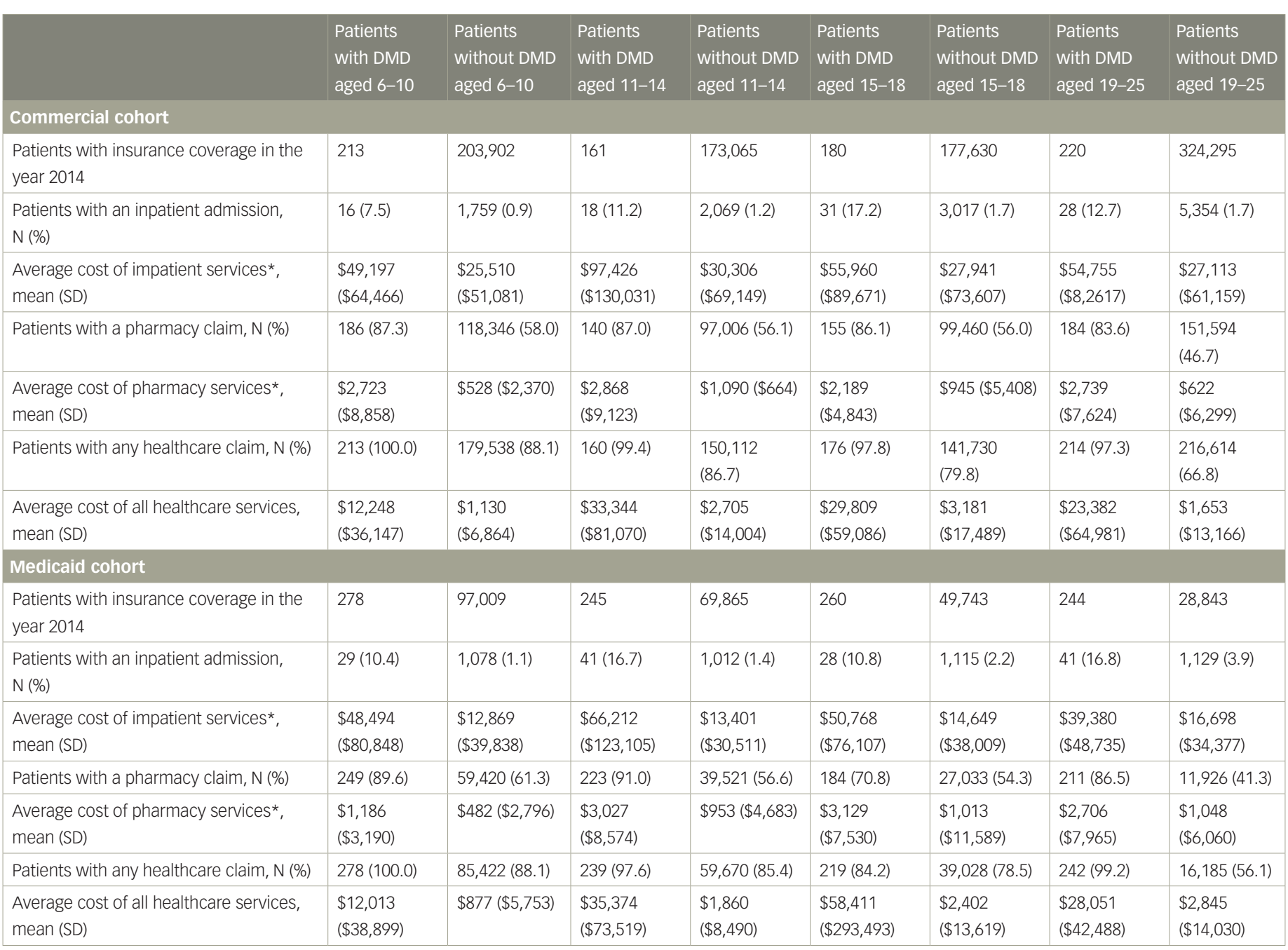

*Payments were calculated as average payment per patient among those patients with a claim in the specified area in the calendar year 2014 . Patient counts are reported as 'N (\%)', averages are reported as 'mean (SD)'. DMD = Duchenne muscular dystrophy; $N=$ number of patients; $S D=$ standard deviation.

Compared with the control group of patients without DMD, there was a higher utilization of antibiotics in the DMD group; unsurprising based on the challenges patients with DMD have in airway clearance. Loss of respiratory function puts patients at risk for severe respiratory complications; antibiotics used to treat the underlying impaired airway clearance can delay this loss of function. ${ }^{17}$ This is, in part, related to the increase in the percentage of patients having a claim for hospital admission increasing from $8.3 \%$ with DMD versus $1.2 \%$ without DMD in the 6-10 age group, to $20.9 \%$ with DMD versus $4.4 \%$ without DMD in the 19-25 age group (Medicaid cohort).

While the average healthcare cost was highest in the 11-14 year old age group, hospital admissions were most frequent in the older age groups. Therefore, an alternate explanation for this increased cost is needed. One explanation is introduction of equipment, with the most likely options as costs of wheelchairs or other durable medical equipment, such as airway clearance devices or non-invasive ventilators. Wheelchair claims did not increase for patients in the 15-18 year old cohort compared to the 11-14 year old cohort, which is not consistent with the increased cost data seen. Therefore, the more likely durable medical equipment cost would be introduction of airway clearance devices, which would be in line with recent standards of care. ${ }^{10-12}$ Previously, Larkindale et al. estimated mean annual DMD costs at $\$ 24,122$ for commercial cohorts; ${ }^{7}$ while this does not drastically differ from our findings, patients were not stratified by age and therefore does not display the increasing costs pattern with disease progression. The present study shows lower costs in the youngest age group, with increased costs in older patients. More research is needed to accurately identify cost-driving factors in patients with DMD that can explain the cost discrepancy among age groups.

Certain limitations of the study design are inherent in any retrospective, administrativeclaims-based cohortstudy. Identifying patientswithDMDbased on the presence of ICD-9-CM diagnosis codes recorded on medical claims may be imprecise, particularly considering the current study cohort. A specific ICD-9-CM diagnosis code does not exist for DMD and therefore male patients with other muscular dystrophies could have been included in these analyses; a number of restrictions were used during patient selection to estimate the DMD population including age and gender restrictions. Our results may underestimate costs, as DMD may be under coded. Only those prescriptions that have been filled are available in our database. 
This analysis only captures claims submitted during the year following diagnosis, and is not reflective of claims that may have been submitted prior, such as prior medical device claims (i.e., wheelchairs). In addition, patients in the study have healthcare coverage, either through commercial insurance, Medicare, or Medicaid, and thus, findings may not be generalizable to patients with DMD who are covered under other types of insurance or are uninsured.
As treatment options for patients with DMD continue to improve with medical advances, extending patient life expectancy will allow for the ability to accurately describe the real-world utilization and outcomes of these treatments. The cumulative cost of these medications and equipment is substantially higher than in the unaffected population and clearly represents a large financial burden that goes along with the burden on quality of life of the patient with DMD. $\square$
1. Yiu EM, Kornberg AJ. Duchenne muscular dystrophy. Neurol India. 2008; $56: 236-47$.

2. Thayer $\mathrm{S}$, Bell $\mathrm{C}, \mathrm{MCD}$ (n)ald $\mathrm{C}$. The direct cost of managing a rare disease: assessing medical and pharmacy costs associated with duchenne muscular dystrophy in the United States. J Manag Care Spec Pharm. 2017;23:633-41.

3. Relizani K, Griffith G, Echevarria L, et al. Efficacy and safety profile of tricyclo-DNA antisense oligonucleotides in Duchenne muscular dystrophy mouse model. Mol Ther Nucleic Acids. 2017;8:144-57.

4. Romitti P, Zhu Y, Puzhankara S, et al. Prevalence of Duchenne and Becker muscular dystrophies in the United States. Pediatrics. 2015;135:513-21.

5. Ryder S, Leadley R, Armstrong N, et al. The burden, epidemiology, costs and treatment for Duchenne muscular dystrophy: an evidence review. Orphanet J Rare Dis. 2017;12:79.

6. The Cleveland Clinic. Muscular Dystrophy, 2015. Available at: https://my.clevelandclinic.org/health/articles/muscular-dystrophy (accessed May 31, 2018).
7. Larkindale J, Yang W, Hogan PF, et al. Cost of illness for neuromuscular diseases in the United States. Muscle Nerve. 2014:49:431-8.

8. Short M, Goetzel, R, Pei X, et al. How accurate are self-reports? An analysis of self-reported healthcare utilization and absence when compared to administrative data. J Occup Environ Med. 2009;51:786-96.

9. Miller L, Romitti P, Cunniff C, et al. The Muscular Dystrophy Surveillance Tracking and Research Network (MD STARnet): surveillance methodology. Birth Defects Res A Clin Mol Teratol. 2006;76:793-7.

10. Hull J, Aniapravan R, Chan E, et al. British Thoracic Society guideline for respiratory management of children with neuromuscular weakness. Thorax. 2012;67(Suppl 1):i1-40.

11. Finder J, Mayer OH, Sheehan D, et al. Pulmonary endpoints in Duchenne muscular dystrophy. A workshop summary. Am J Respir Crit Care Med. 2017;196:512-9.

12. Birnkrant $D$, Bushby $K$, Amin $R$, et al. The respiratory management of patients with Duchenne muscular dystrophy: A DMD Care
Considerations Working Group specialty article. Pediatr Pulmonol. 2010;45:739-48.

13. Kang SW, Kang YS, Moon JH, Yoo TW. Assisted cough and pulmonary compliance in patients with Duchenne muscular dystrophy. Yonsei Med J. 2005:46:233-8.

14. Edward Oppenheimer for Parent Project Muscular Dystrophy Assistive Devices for Coughing. Available at: www. parentprojectmd.org/care/care-guidelines/by-area/care-for-lungmuscles/assistive-devices-for-coughing/ (accessed May 31, 2018)

15. LoMauro A, D'Angelo M, Aliverti A. Assessment and management of respiratory function in patients with Duchenne muscular dystrophy: current and emerging options. Ther Clin Risk Manag. 2015:11:1475-88.

16. MCKim D, Griller N, LeBlanc C, et al. Twenty-four hour noninvasive ventilation in Duchenne muscular dystrophy: a safe alternative to tracheostomy. Can Respir J. 2013;20:5-9.

17. McDonald $\mathrm{C}$, Meier T, Voit T, et al. Idebenone reduces respiratory complications in patients with Duchenne muscular dystrophy. Neuromuscul Disord. 2016:26:473-80. 